\title{
Conditioning in Limulus: Comment on Wasserman and Patton
} WALTER MAKOUS, University of
Washington, Seattle, Wash. 98105

The purpose of this comment is to alert readers to the inconclusiveness of all reports of conditioning in the horseshoe crab, Limulus polyphemus, to date (Makous, 1969; Smith \& Baker, 1960; Wasserman \& Patton, 1969), two of which appeared recently in this Journal. The basic paradigm consists of presenting light (the CS) for $10 \mathrm{sec}$, the last second of which coincides with shock (the US). Wasserman and Patton omitted the shock if the animal responded during the $\mathrm{CS}$, which permits the label of avoidance conditioning to be attached to their procedure.

Subtetanic shock or any other noxious stimulus, as well as many novel stimuli, elicit a downward movement of the tail that orients the animal in a position that facilitates burrowing into the mud at the bottom of the waters Limulus inhabits. The use of these downward movements of the tail for burrowing and ultimate concealment was described a century ago in a delightful paper on the natural history of Limulus by the Rev. Lockwood (1870). These downward movements can be detected during conditioning experiments by use of a special harness described by Wasserman and Patton, by isometric force transducers, by electrical potentials generated in the muscles attached to the tail, and, if the animal is not held down too rigidly, by direct visual observation. Shock and other noxious stimuli may elicit, in addition, general struggling movements that involve the tail in movements in all possible directions; and tetanic shock, such as was used by Wasserman and Patton and perhaps by Smith and Baker, alters the response in ways that depend upon the parameters of the shock and how it is applied.

Wasserman and Patton have reported, in an unpublished paper sent to me in response to an earlier draft of this comment, that some naive animals reliably make downward movements of the tail in response to an overhead light such as serves as the $C S$ in these conditioning experiments. Because of the function of these movements in burrowing, these unconditioned responses to light can be considered part of a negative phototropic response. Both positive and negative phototropisms have frequently been reported in Limulus (Cole, 1922, 1923 Loeb, 1893; Makous, 1969; Northrup \& Loeb, 1923; von Campenhausen, 1967; Waterman, 1953; Wolf \& Zerrahn-Wolf, 1937), and there is evidence that shock increases the strength of the phototropism (Makous, 1969).

The response to be conditioned in all these experiments, then, is a natural part of the animal's escape reaction that may be potentiated by shock. Wasserman and Patton pointed out, in their unpublished paper, that Smith and Baker's demonstration of conditioning is not conclusive, because their controls do not completely eliminate the possibility of pseudoconditioning. Wasserman and Patton's report is not conclusive, for it was a preliminary report containing no controls against pseudoconditioning at all. Further, their attempts to extinguish the response failed, and their US, which passed a current 500 times that necessary to elicit a response, is likely to have sensitized the phototropic response. The last report to be discussed (Makous, 1969) consisted of four parts. The first part, in which operant conditioning was attempted, showed no conditioning. The second part was a pilot experiment that included an exact replication of Smith and Baker's experiment, but its results are inconclusive, for only two animals were used, and there were no controls against pseudoconditioning. The The third part included the appropriate controls against pseudoconditioning (see Rescorla, 1967), in which the control group received as many presentations of the CS and US as the conditioned groups but at random times with respect to one another; but no conditioning could be demonstrated in this experiment. In the last part, which consisted of avoidance conditioning in a situation similar to a shuttlebox, conditioning occurred in the sense that the animals avoided shock often enough over a very large number of trials that explanations based on chance were extremely unlikely, and the experiment was designed so that phototropisms could not account for the avoidances. But the proportion of trials on which the animals responded appropriately was so small (less than $2 \%$ ) that the results are difficult to interpret.

Thus, in spite of Wasserman \& Patton's recent paper (1969), a conclusive demonstration of conditioning in Limulus is still lacking, and Smith \& Baker's (1960) report remains as the most convincing demonstration to date.

\section{REFERENCES}

COLE, W. H. Circus movements of Limulus and the tropism theory. Journal of General Physiology, 1922, 5, 417-426.

COLE, W. H. Effect of laboratory age upon the phototropic reactions of Limulus. Journal of General Phy siology, 1923, 6, 295-297.

LOCKWOOD, S. The horse foot crab. The American Naturalist, 1870, 4, 257-274.

LOEB, J. Über kuenstliche Umwandlung positiv heliotropischer Thiere in negativ heliotropische und umgekehrt. Archiv für die Gesamte Physiologie, 1893, 54, 81-107.

MAKOUS, W. L. Conditioning in the horseshoe crab. Psychonomic Science, 1969, 14, 4-6.

NORTHRUP, J. H., \& LOEB, J. The photochemical basis of animal heliotropism. Journal of General Physiology, 1923, 5, 581-595.

RESCORLA, R. A. Pavlovian conditioning and its proper control procedures. Psychological Review, 1967, 74, 71-80.

SMITH, J. C., \& BAKER, H. D. Conditioning in the horseshoe crab. Journal of Comparative \& Physiological Psychology, 1960, 53, 279-281.

von CAMPENHAUSEN, $C$. The ability of Limulus to see visual patterns. Journal of Experimental Biology, 1967, 46, 557-570.

WASSERMAN, G. S., \& PATTON, D. G. Avoidance conditioning in Limulus. Psychonomic Science, 1969, 15, 143.

WATERMAN, T. H. Action potentials from an arthropod ocellus: The median eye of Limulus. Proceedings of the National Academy of Science, 1953, 39, 687-694.

WOLF, E., \& ZERRAHN-WOLF, G. Reactions of Limulus to illuminated fields of different areas and flicker frequency. Journal of General Physiology, 1937, 20, 767-776. 